\title{
The Role of US Contrast Agents in US-Guided Biopsy of Focal Liver Lesions: A Pictorial Review
}

\section{(ㄷ) (i) ()ㅇ $\Theta$}

\author{
Authors \\ Torben Lorentzen ${ }^{1}$, Christian Pallson Nolsoe ${ }^{2}$
}

\begin{abstract}
US-guided percutaneous biopsy of focal liver lesions (FLL) is a classic interventional procedure performed by almost all radiology units. Typically, an incidental focal finding on US or a focal indeterminate lesion diagnosed on CT, MRI or PET/CT is referred for US-guided biopsy for final diagnosis. The introduction of microbubble US contrast agents has overcome some of the limitations of standard US in diagnosing FLLs by displaying the microvasculature together with the US morphology, which has increased both the sensitivity and the specificity. The combination of CEUS and intervention is facilitated by newer US equipment providing split-screen mode, which displays the CEUS mode alongside the standard US mode simultaneously on a single monitor. The puncture line is displayed in both modes as well as on the monitor. The interventional device (i.e., biopsy needle) is typically best visualized in the standard US mode, while the characteristic tissue pattern in an FLL is typically best visualized in CEUS mode. There are 3 main categories in which CEUS has an impact on US-guided biopsy of FLLs: • CEUS improves the visualization of FLLs - CEUS improves the quality of the biopsy specimen from an FLL - CEUS reduces the need for US-guided biopsy of an FLL In the two first categories, CEUS is utilized simultaneously with US-guided biopsy to ensure correct needle targeting. In the last category, US-guided biopsy of the FLL becomes superfluous as a result of the CEUS examination.
\end{abstract}

\section{Introduction}

Ultrasound (US) imaging is an unsurpassed guide for interventional procedures first and foremost because it is in real time. In addition, it is rapid and convenient, all puncture directions are possible and it allows continuous visualization of the needle tip during needle insertion. There is no ionizing radiation and the equipment is mobile and relatively inexpensive. Because of these advantages, US-guided interventional procedures have achieved widespread use [1,2].

US-guided percutaneous biopsy of focal liver lesions (FLLs) is a classic interventional procedure performed by almost all radiology units. Typically, an incidental focal finding on US or a focal indeterminate lesion diagnosed on CT, MRI or PET/CT is referred for contrast-en- hanced US (CEUS). If still indeterminate, US-guided biopsy might be considered for the final diagnosis. With a $1.2 \mathrm{~mm}$ (18 gauge) automatic cutting needle, a histologic tissue core can be obtained and gives the pathologist optimal conditions for further tissue processing including specialized oncologic techniques (immune-histochemical analysis). Fine needle aspiration (cytology) of FLLs is often sufficient to demonstrate malignancy but insufficient to assess specific tumor markers and perform gene analysis [3].

The development of microbubble US contrast agents has overcome some of the limitations of standard US for FLLs and enables the display of the parenchymal perfusion and microvasculature. The enhancement patterns in an FLL can be studied over time dur- 
ing all vascular phases (arterial, portal venous and late phases), in a similar fashion to contrast-enhanced CT and MRI but in real time and under the full control of the US operator [4]. Several studies have demonstrated that contrast-enhanced US (CEUS) increases sensitivity and specificity in diagnosing FLLs to the same level that can be achieved by contrast-enhanced CT and MRI [5, 6]. Furthermore, allergic and severe reactions induced by CEUS are rare [7]. There are a few limitations when using CEUS for diagnosing FLLs, such as deeply located lesions and advanced fatty liver.

The combination of US contrast agents and intervention is facilitated by newer US equipment, providing the split-screen mode, which displays the CEUS mode alongside the standard US mode simultaneously on a single monitor. The puncture line is displayed in both modes as well on the monitor. The interventional device (i. e. biopsy needle) is typically best visualized in the standard US mode, whereas the characteristic tissue pattern in an FLL is typically best visualized in CEUS mode.

This pictorial review focuses on the impact of CEUS on US-guided biopsy of FLLs [8]. It is based on a literature search of PubMed performed in June 2018. The amount of scientific work in this field is still limited, but a clear tendency and some obvious advantages with CEUS-guided biopsy will be demonstrated. Several illustrative cases (figures and links to videos) will be presented as well. In our opinion, there are 3 main categories that show the impact of CEUS on US-guided biopsy of FLLs:
- CEUS improves the visualization of FLLs

- CEUS improves the quality of the biopsy specimen from an FLL

- CEUS reduces the need for US-guided biopsy of an FLL

In the two first categories, CEUS is utilized simultaneously with US-guided biopsy to ensure correct needle targeting which, in a recent multi-center study, was needed in $2.6 \%$ of all US-guided biopsies of FLLs [9]. In the last category, US-guided biopsy of the FLL becomes superfluous as a result of the CEUS examination.

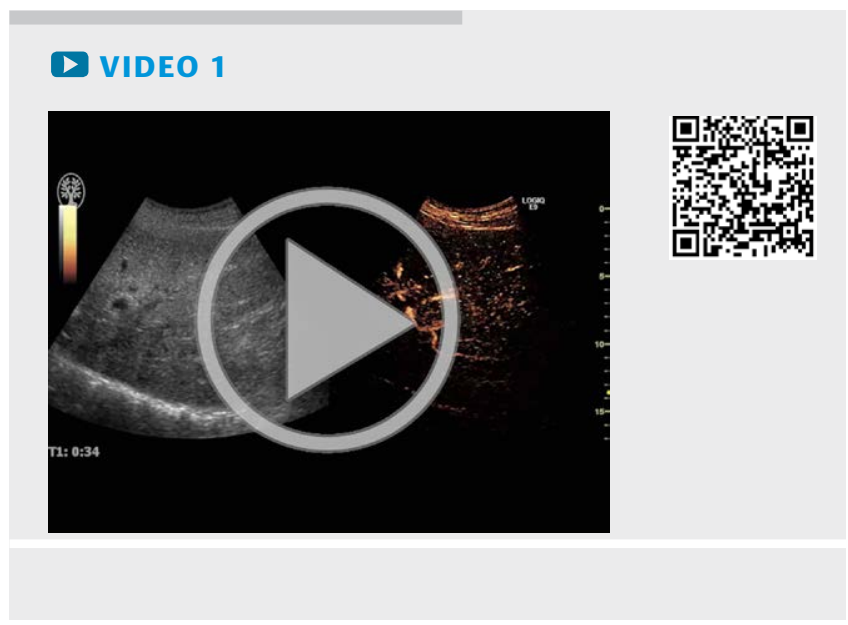

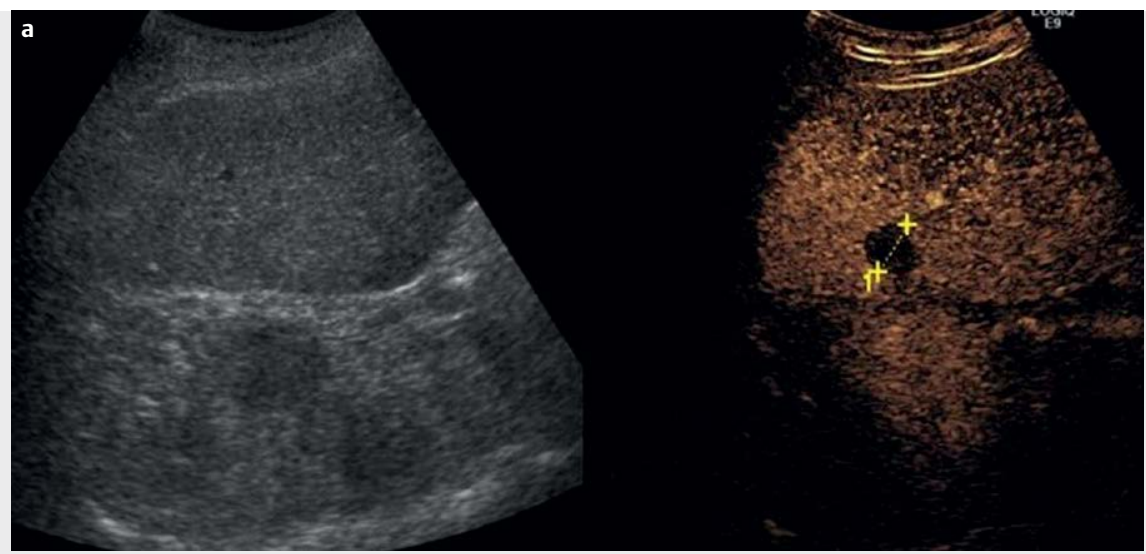

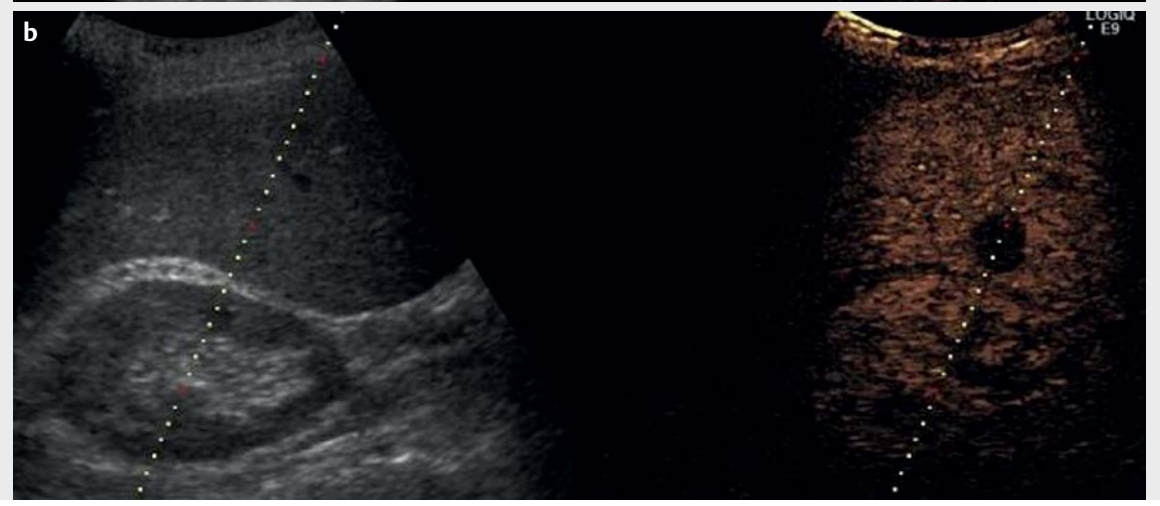

-Fig. 1 Improved visualization of liver metastasis after CEUS. Patient with disseminated breast cancer and liver metastases seen on recent PET/CT. On fundamental US (image to the left), the metastasis cannot be seen. In CEUS mode (image to the right) a clearly depicted lesion (metastasis) is seen between the 2 calipers located close to the right kidney (Fig 1a). Puncture line has been applied; still the metastasis is only visible in CEUS mode (Fig. 1b). 

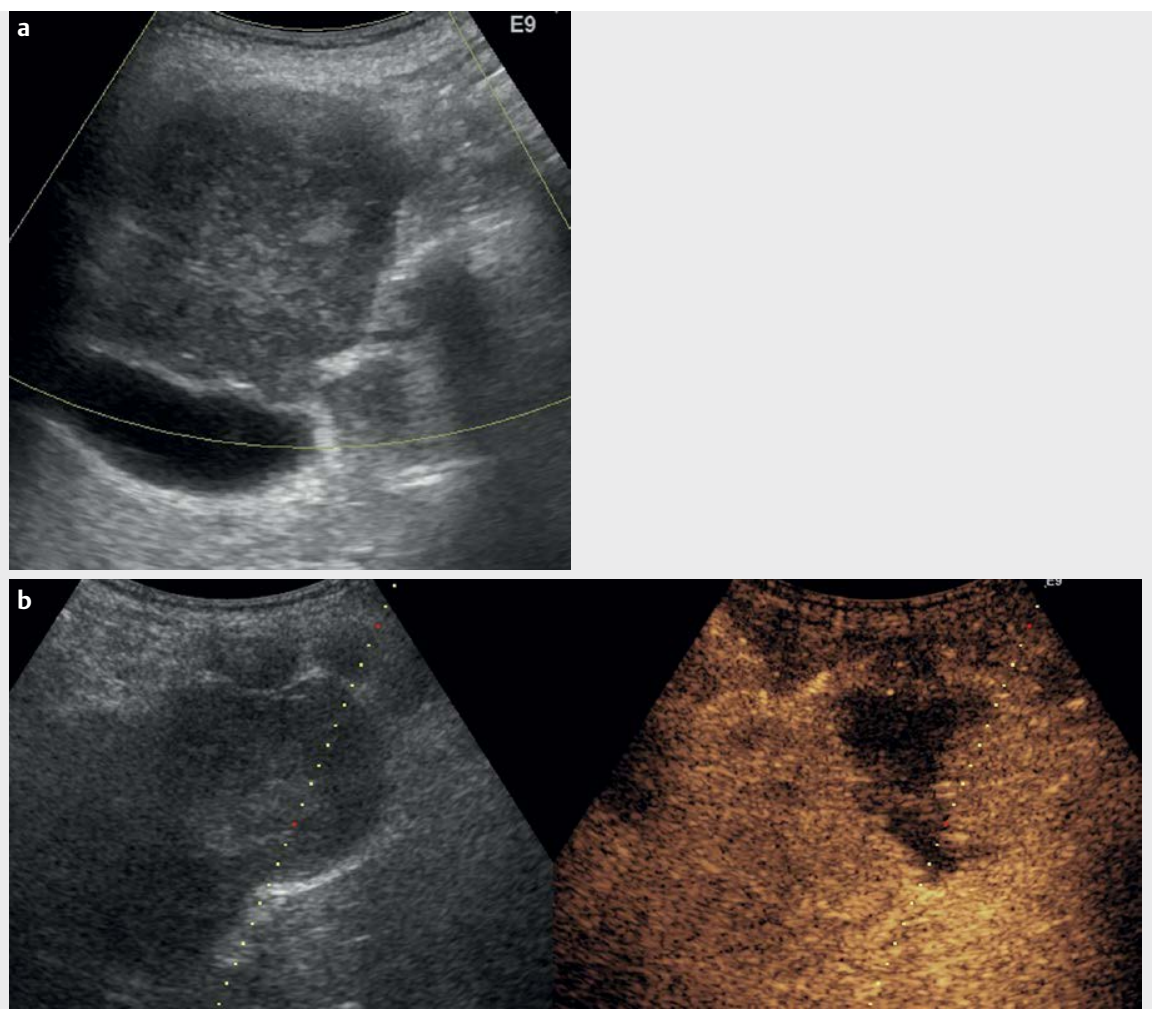

-Fig. 2 CEUS improves the quality of the biopsy specimen from FLLs. In a patient with disseminated malignant melanoma, a new biopsy from liver metastasis was requested by the oncologists. On fundamental US, a $6 \mathrm{~cm}$ lesion is seen in the left liver lobe (segment 4) close to the gall bladder (Fig. 2a). After CEUS, most of the liver metastasis is seen to be necrotic and avascular, and only the right part of the tumor is vascularized (where the puncture line has been applied just before percutaneous biopsy guided by CEUS) (Fig. 2b).

\section{VIDEO 2}

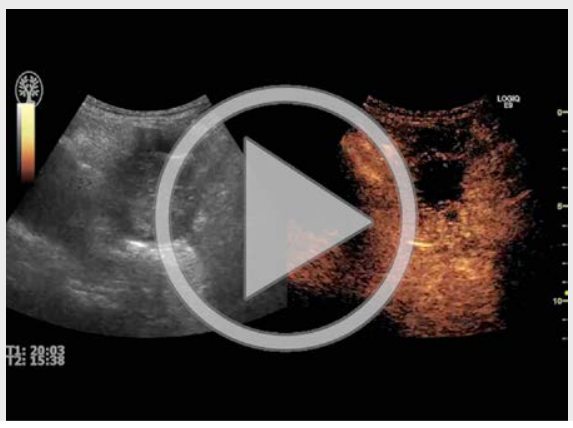

\section{Review and Discussion}

\section{CEUS improves the visualization of FLLs}

Most FLLs are clearly visualized on standard B-mode US and a biopsy can therefore be performed successfully with guidance by standard US in most cases. However, some FLLs might be difficult or impossible to see on standard US due to a lack of tissue contrast between the lesion and the surrounding normal liver tissue, thus an isoechoic lesion. These patients are often referred after a suspicious finding on other image modalities (CT, MRI or PET/CT), and a US-guided biopsy is requested for achieving the final diagnosis. In such cases, CEUS may be helpful in 2 different ways: 1. the suspicious lesion seen on previous imaging may turn from invisible or inconspicuous on standard US to "clearly visualized" on CEUS imaging; and 2. Additional FLLs that are potentially more easily accessible for biopsy may be visualized and biopsied under CEUS visualization and guidance.

In 2004, Schlottmann et al. in a small series of 12 patients with FLLs that were invisible on standard US demonstrated that CEUS made all (100\%) of the lesions visible. The following CEUS-guided biopsy gave the final diagnosis in 11 (92\%) of the 12 cases [10]. In a recent retrospective study by Partovi at al., 26 patients with poorly visualized or invisible FLLs on standard US were referred for CEUS-guided biopsy as an alternative to CT-guided biopsy. In 23 (88\%) of the patients, CEUS-guided biopsy was reported to be successful [11]. A prospective nonrandomized study by Yoon et al. included 44 patients with FLLs that were not able to be confidently located for biopsy on standard US. CEUS-guided biopsy was possible in 39 patients (89\%), and the biopsy yielded the final diagnosis in 38 patients (86\%) [12]. In a prospective study by Park et al., 66 patients were enrolled for US-guided biopsy or US-guided RF ablation. CEUS increased the detection rate of FLLs from $77 \%$ (standard US) to $92 \%$ and a success rate of CEUS-guided biopsy of 95\% was reported [13]. Finally, Sparchez et al. reported a prospective study with 171 patients with FLLs (seen on CT or MRI) referred for US-guided biopsy and randomized into 2 groups: One group had liver biopsy guidance by standard US and the other group underwent CEUS-guided biopsy. A correct pathological diagnosis could be reached in $97 \%$ of the CEUS group and $81 \%$ of the standard US group. 

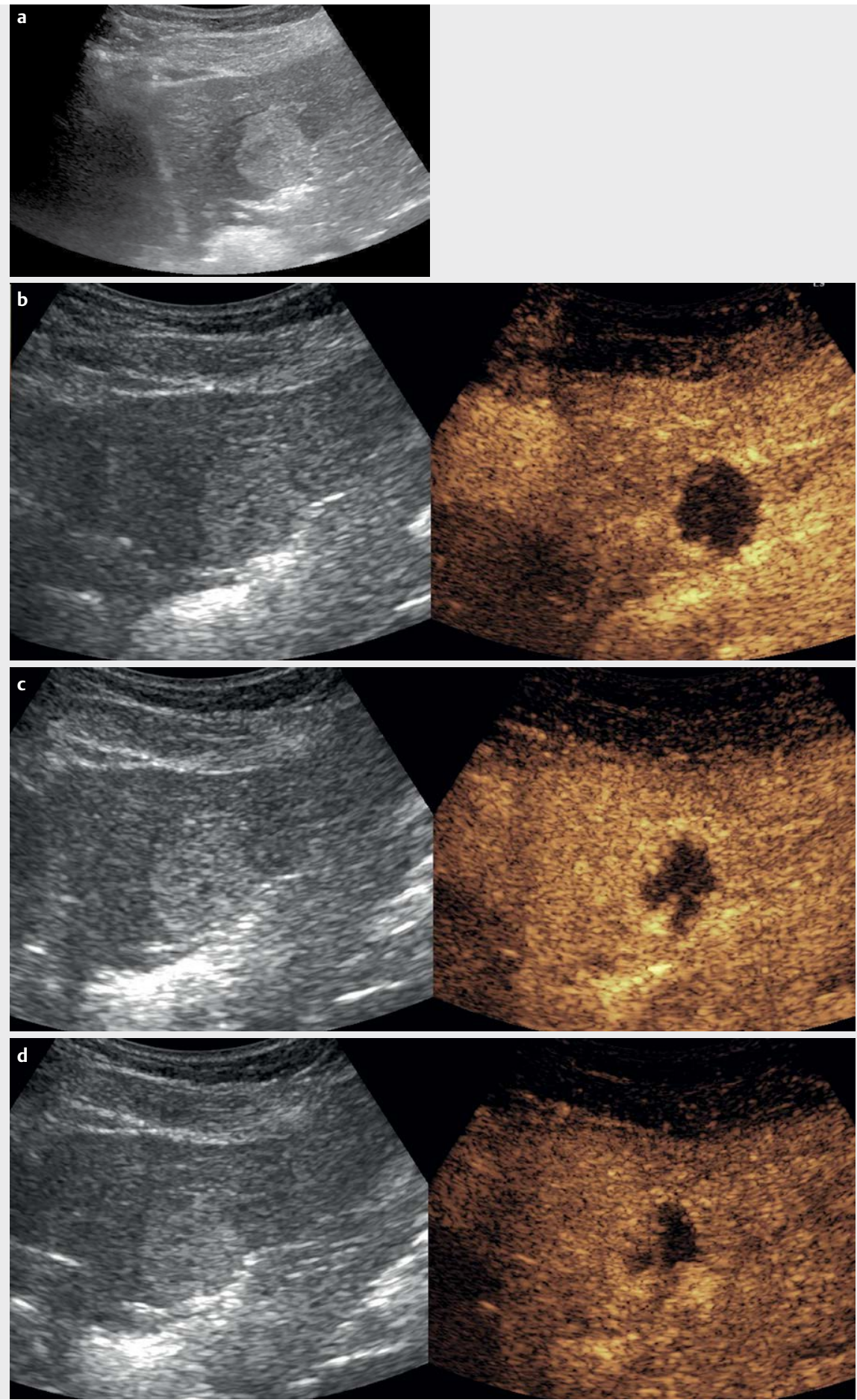

- Fig. 3 Typical hemangioma on US confirmed with CEUS. Incidental finding of a $3 \mathrm{~cm}$ echogenic, homogeneous and well demarcated mass in the left liver lobe (Fig. 3a). In CEUS mode the monitor shows fundamental US mode to the left and contrast US mode to the right. In the arterial phase (after 20s), characteristic rim enhancement is demonstrated around the hemangioma (Fig. 3b). After $60 \mathrm{~s}$ (late phase), the rim enhancement is progressing in a centripetal direction (Fig. 3c). After $120 \mathrm{~s}$, the rim enhancement is further progressing in a centripetal direction to a partial fill-in (Fig. 3d). Thus, this is an example of a CEUS examination that made US-guided biopsy superfluous.

Especially in patients with cirrhosis, CEUS improved tumor visualization significantly and thereby the diagnostic accuracy [14].

The two latter studies confirm that standard US is most often sufficient as the primary biopsy-guiding modality and CEUS guidance can be reserved for cases with poor or no visibility on stand- ard US. In cases in which CEUS mode does not provide satisfying visualization of an FLL, other US techniques, such as fusion imaging or elastography, may be considered $[15,16]$. Alternatively, biopsy of an FLL that is "invisible" on CEUS can be performed under CT or MRI guidance.( $\triangleright$ Fig. 1, Video 1$)$ 
$\checkmark$ VIDEO 3

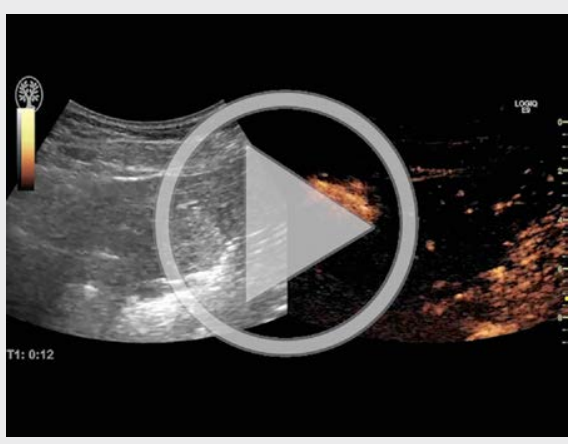

$\checkmark$ VIDEO 4

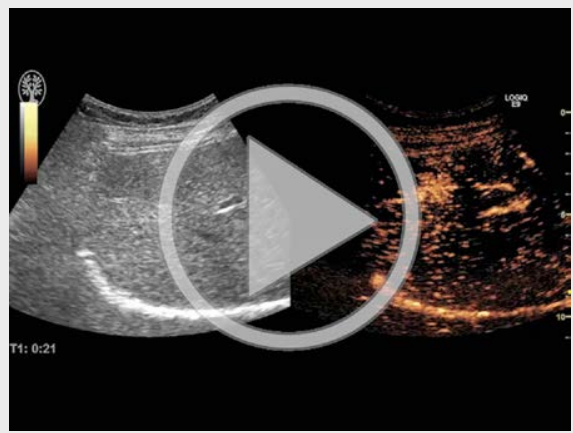

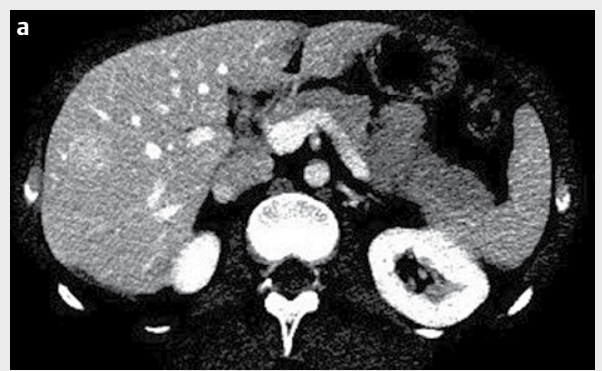
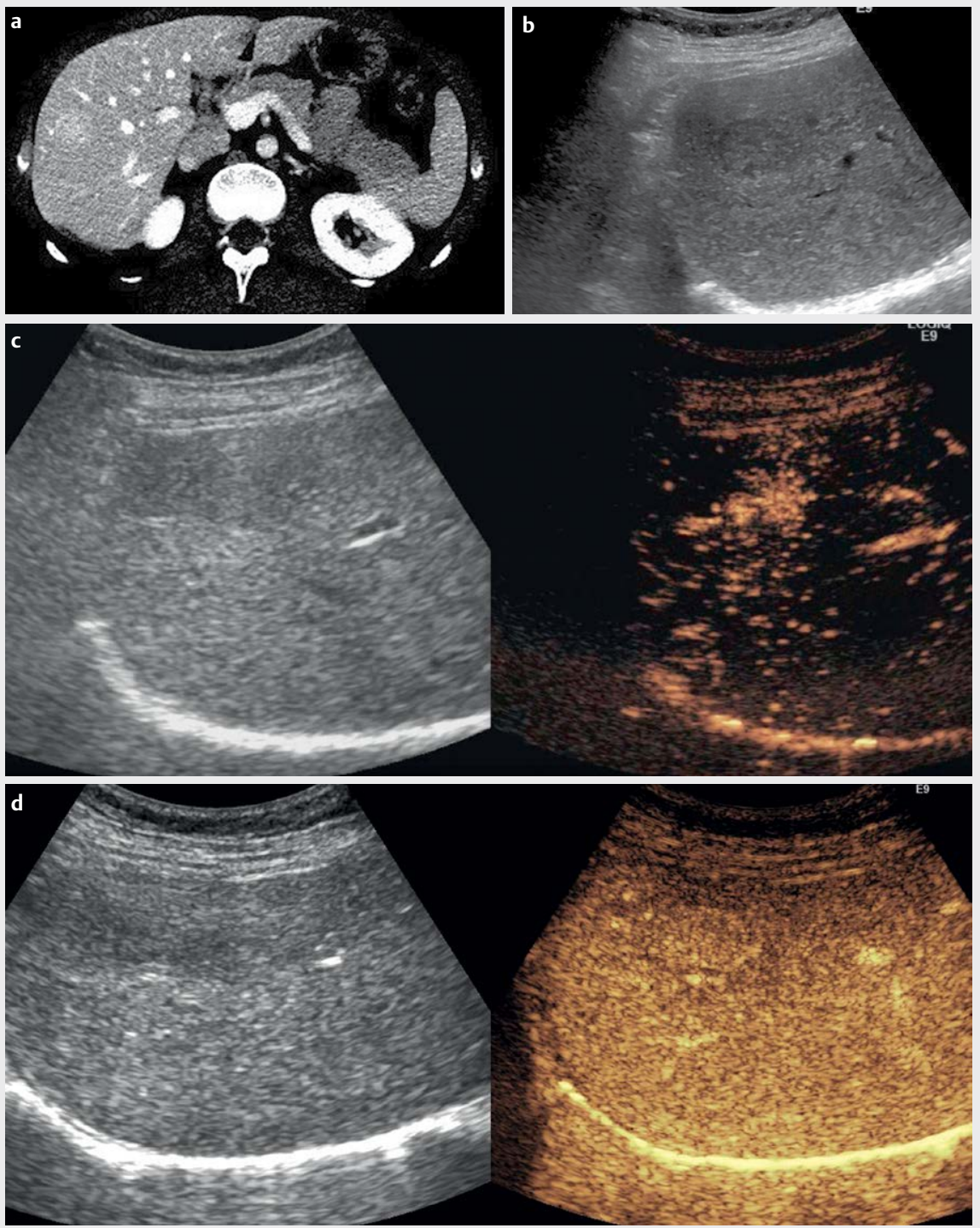

-Fig. 4 Typical FNH demonstrated on CEUS. Primarily identified on CT as a focal indeterminate hypervascular lesion in the right liver lobe (Fig. 4a). On fundamental US, the lesion was slightly hypoechoic (Fig. 4b). In CEUS mode, in the arterial phase (after $10 \mathrm{~s}$ ) a characteristic spoke-wheel pattern is seen (Fig. 4c). In the late phase, the FNH becomes almost isovascular on CEUS (Fig. 4d). Thus, this is an example of a CEUS examination that made US-guided biopsy superfluous. 


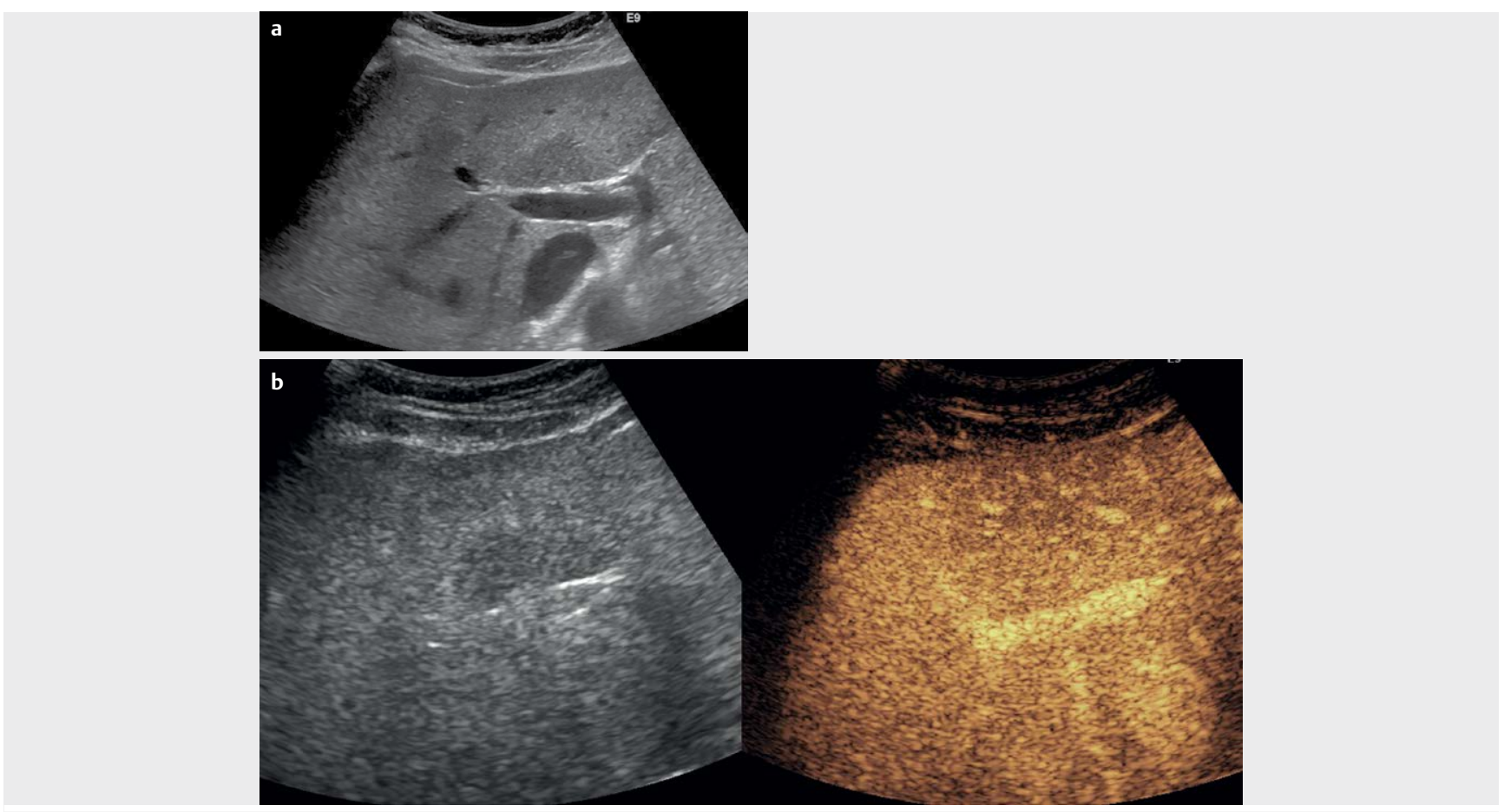

- Fig. 5 Typical focal fatty change in B mode and CEUS mode. In B mode, a hypoechoic lesion with a patchy, geographic pattern appearance is located anterior to the portal vein (Fig. 5a). In CEUS mode, in the late phase (after $60 \mathrm{~s}$ ) the lesion in contrast mode (image to the right) is isovascular - typical for focal fatty change (Fig. 5b). Thus, this is an example of a CEUS examination that made US-guided biopsy superfluous.

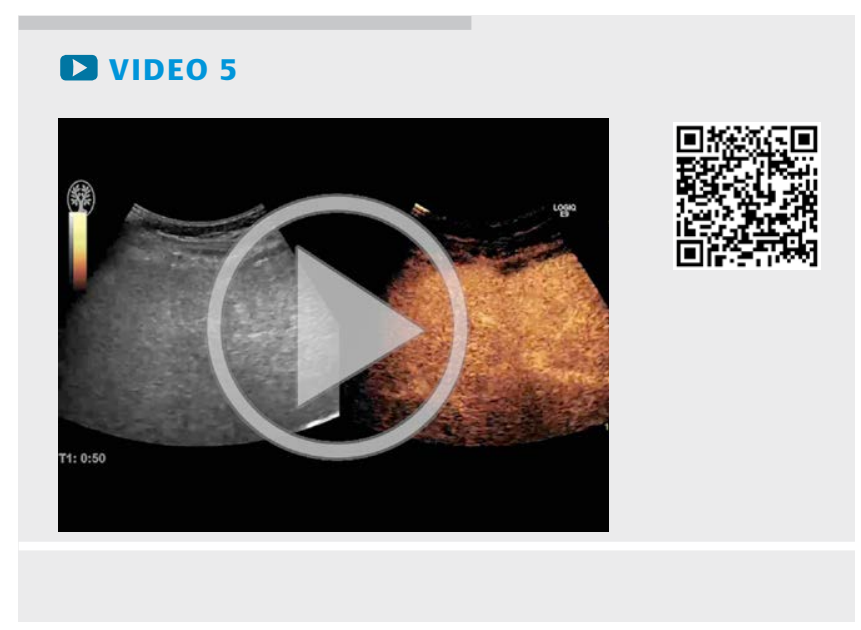

\section{CEUS improves the quality of the biopsy specimen from an FLL}

Sometimes, the pathology report from an FLL biopsy may simply read as necrotic or insufficient material. Especially large tumors (both hepatic and non-hepatic) tend to contain necrotic and liquefied areas - often located centrally due to perfusion insufficiency. A repeated biopsy procedure is then warranted to obtain decisive and viable material, and increased attention is paid to avoid sampling from necrotic areas within the lesion. With CEUS, tissue vascularization and thereby viability can be evaluated effectively. By directing the biopsy needle toward contrast-enhanced areas with- in the target lesion, inadvertent sampling from necrotic parts of the tumor can be reduced.

In a prospective study, Wu et al. included 186 patients with uncertain FLLs. Patients were randomized to a standard US or a CEUS pre-biopsy examination looking for tumor enhancement and viability. The diagnostic accuracy of the initial biopsy was significantly higher in the CEUS group than in the standard US group (95\% versus $87 \%$ ). It should be noted that all biopsies were performed guided solely by standard US. An even higher diagnostic accuracy was reported in patients with small FLLs $<2 \mathrm{~cm}$, which is surprising since tumor necrosis is often associated with larger tumors. However, we believe that the higher diagnostic accuracy was a result of improved visualization by CEUS [17]. In a prospective study, Eso et al. reported significantly fewer cases of insufficient biopsy material from FLLs when the biopsy was guided by CEUS rather than by standard US [18]. Finally, the prospective study by Sparchez already mentioned above reported a significant increase in diagnostic accuracy when large FLLs $>6 \mathrm{~cm}$ underwent CEUS-guided biopsy probably due to a reduced number of insufficient biopsies containing necrotic material [14].

Improvement of biopsy quality due to the use of CEUS guidance has also been demonstrated in non-hepatic tumors in several papers [19-21]. There is no evidence to support and justify the routine use of US contrast agents in all US-guided biopsies of FLLs because of cost and time. However, we recommend CEUS guidance when a re-biopsy is requested due to either an insufficient initial biopsy with necrotic material or insufficient visualization, which might be relevant in a small percentage of all biopsies [9]. ( Fig. 2 , Video 2) 

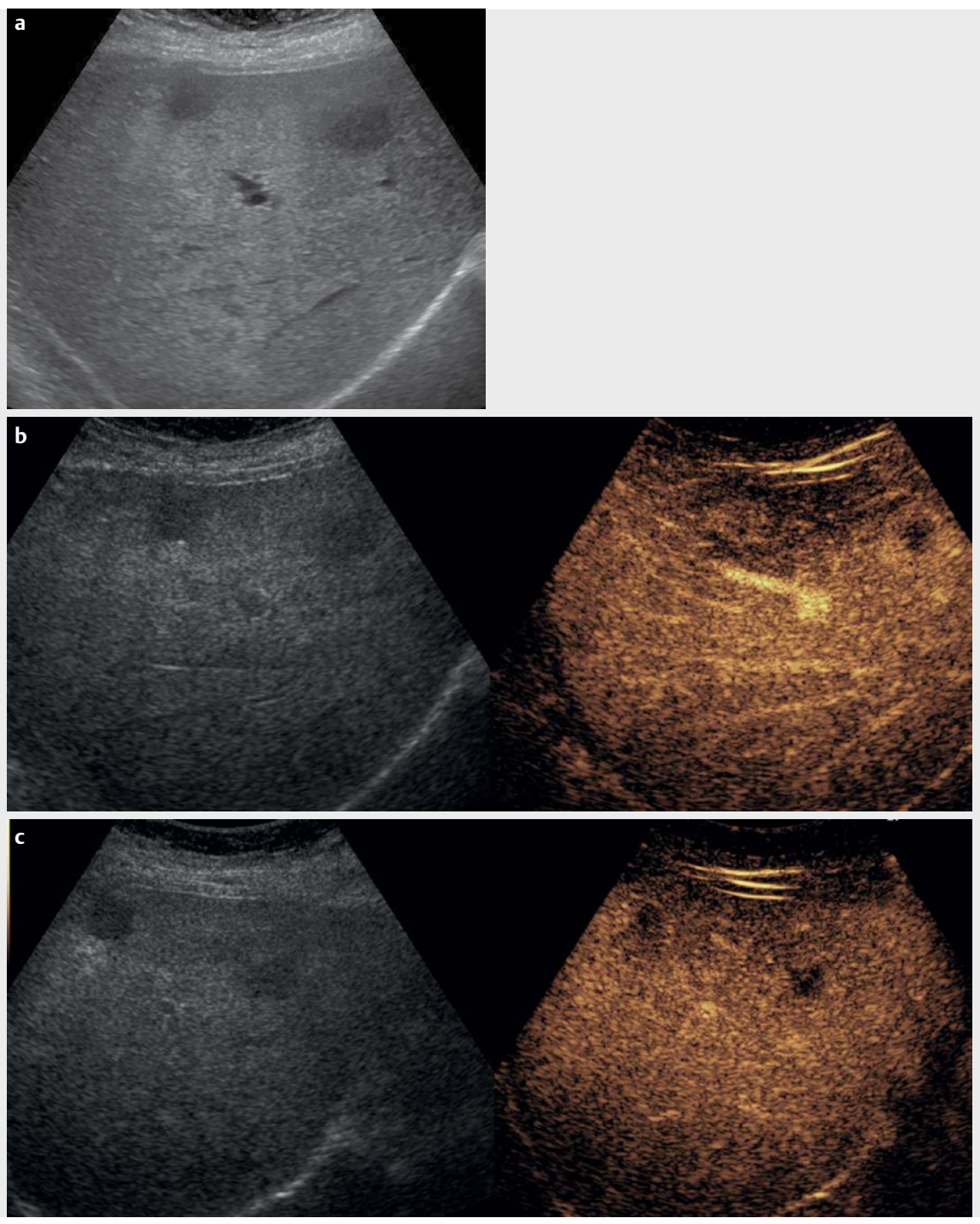

-Fig. 6 Typical liver metastases in B mode and CEUS mode. In B mode, 2 hypoechoic and suspicious lesions of 15-20 mm are visualized in the liver (Fig. 6a). In the arterial phase (after 20 s), the 2 FLLs are hyperenhancing (Fig. 6b). In the late phase (after 1-2 min), the 2 FLLs are hypoenhancing (washout) (Fig. 6c). The finding was confirmed in other imaging modalities and the patient was operated (liver resection) without histologic confirmation.

\section{CEUS reduces the need for US-guided biopsy of an FLL}

Standard US of the liver is sufficient for characterizing many benign FLLs, such as cysts, hyperechoic hemangiomas, typically located focal fat accumulations or fatty sparing. Atypical findings, on the other hand, often require other modalities such as CT, MRI, and $\mathrm{PET} / \mathrm{CT}$ and/or US-guided biopsy to reach a conclusive diagnosis. When it comes to malignant FLLs, US-guided biopsy has been almost a routine procedure to reach the final diagnosis. Percutaneous biopsy of benign and malignant FLLs is still regarded as the gold standard for the final diagnosis. However, the introduction of CEUS has significantly improved the ability to characterize FLLs based on imaging alone, thus giving an almost conclusive and final diagnosis without the need for biopsy and histologic proof. There is now substantial scientific evidence to support this statement in the form of guidelines, systematic reviews and meta-analyses [22-27]. Thus, according to the EFSUMB Guidelines on Interventional Ultrasound [28], the indications for US-guided FLL biopsy are:

- Diagnosis not established by any imaging method

- Lesion immune-histochemical analysis needed for therapy

- Histological assessment needed for a therapeutic decision (e.g. hepatocellular carcinoma vs. cholangiocarcinoma).

Some of the typical characteristics of FLLs seen on CEUS examination are as follows:

\section{Benign FLLs:}

Hemangioma

US finding of an atypical hemangioma requires further imaging, possibly biopsy. CEUS has markedly improved the accurate diagnosis of hemangiomas, which is now possible in about $95 \%$ of cases [5]. The typical CEUS features of a hemangioma are peripheral nodular en- 


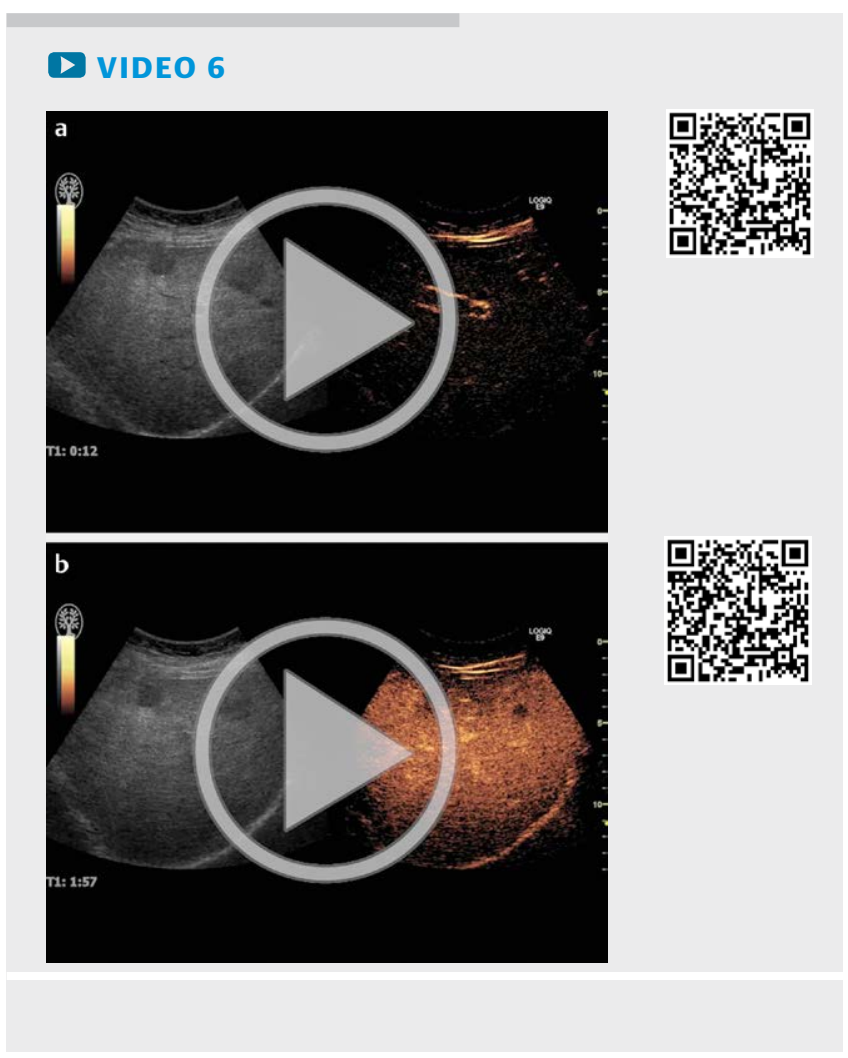

hancement in the arterial phase (the rim sign) to partial or complete fill-in progressing in a centripetal direction ( $\mathbf{F i g . ~ 3 , ~ V i d e o ~} 3$ ). The filling-in lasts from seconds to minutes and is more rapid in smaller lesions. Enhancement is sustained through the late phase [22]. Thrombosed hemangiomas can be confused with malignancies because of the lack of enhancement in all vascular phases in the thrombosed portions, which may be misinterpreted as washout if only the late phase is studied [29].

\section{Focal nodular hyperplasia (FNH)}

On CEUS, FNH typically appears as a hyperenhancing homogeneous lesion in all phases. Hyperenhancement is obvious and usually marked in the arterial phase, with rapid fill-in from the center outwards (70\%), the so-called spoke-wheel pattern or with an eccentric vascular supply (30\%) [30]. During the portal venous and late phases, $\mathrm{FNH}$ may remain slightly hyperenhancing or become isoenhancing and a centrally located scar may be seen. It is hypoenhancing in the late phase [22] ( Fig. 4, Video 4)

Focal fatty change, either fat infiltration or fatty sparing, may simulate masses on standard US. Differential diagnosis is important, especially in patients with underlying malignant disease or with an atypical location of suspected focal fatty changes. On CEUS, focal fatty change shows exactly the same enhancement patterns as the adjacent liver parenchyma in all phases [22,31]. ( Fig. 5, Video 5)

\section{Malignant FLLs:}

Metastases

Liver metastases can be detected and characterized reliably as hypoenhancing lesions during the portal venous and late phases, with very few exceptions. Washout starts early, usually in the portal venous phase, and is marked. Thus, they appear as "black foci" against the background of the uniformly enhanced normal liver parenchyma [22]. ( Fig. 6, Video 6)

\section{Hepatocellular carcinoma (HCC)}

Typical finding is an intense arterial uptake followed by washout of contrast in the venous and late phase. Unfortunately, however, between $50 \%$ and $70 \%$ of well differentiated HCCs -corresponding to approximately $10 \%-15 \%$ of HCCs in the cirrhotic population - may not demonstrate this typical enhancement pattern [22, 32]. Furthermore, overlapping characteristics have been demonstrated in CEUS imaging of HCC and cholangiocarcinoma [33]. Consequently, sole use of CEUS in the detection of HCC and cholangiocarcinoma cannot be recommended and other imaging modalities or ultimately biopsy should be considered.

In our hospital, we now only perform US-guided biopsy of "benign" FLLs if CEUS imaging together with other imaging modalities (CT, MRI, $\mathrm{PET} / \mathrm{CT}$ ) and the clinical history make the lesion appear atypical and suspicious for malignancy. Regarding obviously malignant FLLs, we only perform biopsy by request and after evaluation by a multidisciplinary team. A patient with liver metastases based on imaging and deemed resectable by surgeons will go directly to surgery without biopsy since surgeons prefer to eliminate the small risk of seeding in the puncture tract [34]. Patients with multiple liver metastases are referred to oncologic treatment and the need for metastasis biopsy depends on several factors including the need for immune-histochemical analysis.

\section{Conclusion}

CEUS is a helpful tool when biopsy of FLLs is requested or considered, especially when the lesion is inconspicuous or invisible on standard US and/or when the initial biopsy was insufficient due to necrotic material. Furthermore, CEUS has improved FLL characterization significantly and has thereby made many biopsies of benign and malignant FLLs superfluous.

\section{Conflict of Interest}

No conflict of interest has been declared by the authors.

\section{References}

[1] Lorentzen T, Nolsøe C. Interventional ultrasound - general principles and applications in gastroenterology ASUM. Ultrasound Bulletin 2008; 11: $25-29$

[2] Lorentzen T, Nolsøe CP, Ewertsen C et al. EFSUMB guidelines on interventional ultrasound (INVUS), part I: General aspects (long version). Ultraschall Med 2015; 36: E1-E14

[3] Ghent CN. Percutaneous liver biopsy: Reflections and refinements. Can J Gastroenterol 2006; 20: 75-79

[4] Skjoldbye B, Bachmann Nielsen M, Nolsøe C. Ultrasound contrast agents in diagnosis of focal liver lesions. ASUM Ultrasound Bulletin 2007; 10: 32-34 
[5] Strobel D, Seitz K, Blank W et al. Contrast-enhanced ultrasound for the characterization of focal liver lesions-diagnostic accuracy in clinical practice (DEGUM multicenter trial). Ultraschall Med. 2008; 29 : 499-450

[6] Larsen LP, Rosenkilde M, Christensen $\mathrm{H}$ et al. The value of contrast enhanced ultrasonography in detection of liver metastases from colorectal cancer: A prospective double-blinded study. Eur J Radiol 2007; 62: 302-307

[7] Piscaglia F, Bolondi L. The safety of SonoVue in abdominal applications: Retrospective analysis of 23,188 investigations. Ultrasound Med Biol 2006; 32: 1369-1375

[8] Nolsøe CP, Nolsøe AB, Klubien J et al. Use of ultrasound contrast agents in relation to percutaneous interventional procedures: $A$ systematic review and pictorial essay. J Ultrasound Med 2018; 37: $1305-1324$

[9] Francica G, Meloni MF, de Sio I et al. Biopsy of Liver Target Lesions under Contrast-Enhanced Ultrasound Guidance - A Multi-Center Study. Ultraschall Med. 2017, doi:10.1055/s-0043-122496

[10] Schlottmann K, Klebl F, Zorger $\mathrm{N}$ et al. Contrast-enhanced ultrasound allows for interventions of hepatic lesions which are invisible on conventional B-mode. Z Gastroenterol 2004; 42: 303-310

[11] Partovi S, Lu Z, Kessner R et al. Contrast enhanced ultrasound guided biopsies of liver lesions not visualized on standard B-mode ultrasound-preliminary experience. J Gastrointest Oncol 2017; 8: 1056-1064

[12] Yoon SH, Lee KH, Kim SY et al. Real-time contrast-enhanced ultrasound-guided biopsy of focal hepatic lesions not localised on B-mode ultrasound. Eur Radiol 2010; 20: 2047-2056

[13] Park HS, Kim Y], Yu MH et al. Real-time contrastenhanced sonographically guided biopsy or radiofrequency ablation of focal liver lesions using perflurobutane microbubbles (Sonazoid):Value of Kupffer-phase imaging. J Ultrasound Med 2015; 34: 411-421

[14] Spârchez Z, Radu P, Kacso G et al. Prospective comparison between real time contrast enhanced and conventional ultrasound guidance in percutaneous biopsies of liver tumors. Med Ultrason 2015; 17 : 456-463

[15] Park HJ, Lee MW, Lee MH et al. Fusion imaging-guided percutaneous biopsy of focal hepatic lesions with poor conspicuity on conventional sonography. J Ultrasound Med 2013; 32: 1557-1564

[16] Conti CB, Cavalcoli Fl, Fraquelli Ml et al. Ultrasound elastographic techniques in focal liver lesions. World J Gastroenterol 2016; 22: 2647-2656

[17] Wu W, Chen MH, Yin SS et al. The role of contrast-enhanced sonography of focal liver lesions before percutaneous biopsy. AJR Am J Roentgenol 2006; 187: 752-761

[18] Eso Y, Takai A, Takeda $\mathrm{H}$ et al. Sonazoid-enhanced ultrasonography guidance improves the quality of pathological diagnosis in the biopsy of focal hepatic lesions. Eur J Gastroenterol Hepatol 2016; 28: 1462-1467

[19] De Marchi A, Brach del Prever EM, Linari A et al. Accuracy of coreneedle biopsy after contrast-enhanced ultrasound in soft-tissue tumours. Eur Radiol 2010; 20: 2740-2748
[20] Mao F, Dong Y, ji Z et al. Contrast-enhanced ultrasound guided biopsy of undetermined abdominal lesions: A multidisciplinary decision-making approach. Biomed Res Int 2017; 2017: 8791259

[21] Sparchez Z, Radu P, Kacso G et al. Contrastenhanced ultrasound guided biopsy of superficial thoraco-abdominal and neck lesions: Initial experience in 20 patients. Med Ultrason 2012; 14: 288-293

[22] Claudon M, Dietrich CF, Choi BI et al. Guidelines and good clinical practice recommendations for contrast enhanced ultrasound (CEUS) in the liver-update 2012: A WFUMB-EFSUMB initiative in cooperation with representatives of AFSUMB, AIUM, ASUM, FLAUS and ICUS. Ultrasound Med Biol 2013; 39: 187-210

[23] Friedrich-Rust M, Klopffleisch T, Nierhoff J et al. Contrast-enhanced ultrasound for the differentiation of benign and malignant focal liver lesions: A meta-analysis. Liver Int 2013; 33: 739-755

[24] Guang Y, Xie L, Ding H, Cai A, Huang Y. Diagnosis value of focal liver lesions with SonoVueVR -enhanced ultrasound compared with contrast-enhanced computed tomography and contrastenhanced MRI: A meta-analysis. J Cancer Res Clin Oncol 2011; 137: 1595-1605

[25] Westwood M, Joore M, Grutters ] et al. Contrast-enhanced ultrasound using SonoVueVR (sulphur hexafluoride microbubbles) compared with contrast-enhanced computed tomography and contrastenhanced magnetic resonance imaging for the characterisation of focal liver lesions and detection of liver metastases: A systematic review and cost-effectiveness analysis. Health Technol Assess 2013; 17: 1-243

[26] Xie L, Guang Y, Ding H, Cai A, Huang Y. Diagnostic value of contrast-enhanced ultrasound, computed tomography and magnetic resonance imaging for focal liver lesions: A meta-analysis. Ultrasound Med Biol 2011; 37: 854-861

[27] Nolsøe C, Lorentzen T. International guidelines for contrast-enhanced ultrasonography: Ultrasound imaging in the new millennium. Ultrasonography 2016; 35: 89-103

[28] Sidhu PS, Brabrand K, Cantisani V et al. EFSUMB guidelines on interventional ultrasound (invus), part ii. diagnostic ultrasound-guided interventional procedures (long version). Ultraschall Med. 2015; 36: E15-E35

[29] Dietrich CF, Mertens JC, Braden B et al. Contrast-enhanced ultrasound of histologically proven liver hemangiomas. Hepatology 2007; 45: 1139-1145

[30] Dietrich CF, Schuessler G, Trojan J et al. Differentiation of focal nodular hyperplasia and hepatocellular adenoma by contrast-enhanced ultrasound. Br J Radiol 2005; 78: 704-707

[31] Hirche TO, Ignee A, Hirche $\mathrm{H}$ et al. Evaluation of hepatic steatosis by ultrasound in patients with chronic hepatitis $C$ virus infection. Liver Int 2007; 27: 748-757

[32] Joo I, Choi BI. New paradigm for management of hepatocellular carcinoma by imaging. Liver Cancer 2012; 1: 94-109

[33] Bohle WI, Clemens PU, Heubach T, Zoller WG. Contrast-enhanced ultrasound (CEUS) for differentiating between hepatocellular and cholangiocellular carcinoma. Ultraschall Med. 2012; 33: E191-E195

[34] Chen I, Lorentzen T, Linnemann D et al. Seeding after ultrasound-guided percutaneous biopsy of liver metastases in patients with colorectal or breast cancer. Acta Oncol. 2016; 55: 638-643 